\title{
GENERAL
}

\section{MODELS OF IMPLEMENTATION OF THE FALSIFIED MEDICINES DIRECTIVE IN DIFFERENT DISPENSING SCENARIOS}

\author{
PIOTR MERKS ${ }^{1,2 *}$, URSZULA RELGIONI ${ }^{3}$, NUNO PINTO DE CASTRO ${ }^{4}$, \\ MARIOLA DROZD ${ }^{5}$, DAMIAN ŚWIECCZKOWSKI ${ }^{6}$, MIKOŁAJ ZERHAU ${ }^{7}$, \\ ANNA GAWROŃSKA ${ }^{8}$, ANNA KOWALCZUK ${ }^{9}$, MIŁOSZ JAGUSZEWSKI ${ }^{6}$, \\ DAVID BRINDLEY ${ }^{10}$ and MARTIN HUG ${ }^{11}$
}

${ }^{1}$ Faculty of Medicine, Collegium Medicum, Cardinal Stefan Wyszynski University, Warsaw, Poland ${ }^{2}$ Department of Pharmaceutical Technology, Faculty of Pharmacy,

Collegium Medicum in Bydgoszcz, Poland

${ }^{3}$ Department of Business Administration, Warsaw School of Economics, Poland ${ }^{4}$ Open University Business School, Walton Hall, Kents Hill,

Milton Keynes MK7 6AA, United Kingdom

${ }^{5}$ Department of Ethics and Medical Law, Department of Social Medicine, Inter-faculty, Didactic Center, Medical University of Lublin, Poland

${ }^{6}$ First Department of Cardiology, Medical University of Gdansk, Poland

${ }^{7}$ Dr. Jozef Psarski Masovian Specialist Hospital in Ostroleka, Poland

${ }^{8}$ Lukasiewicz Institute of Logistics and Warehousing,

Standardisation Department, Poznan, Poland

${ }^{9}$ National Institute of Medicines, Warsaw, Poland

${ }^{10}$ UCL Centre for the Advancement of Sustainable Medical Innovation (CASMI),

The University of Oxford, Oxford, United Kingdom

${ }^{11}$ Department of Pharmacy, University of Freiburg, Freiburg, Germany

\begin{abstract}
To minimize the burden and maximize the benefits of the Falsified Medicines Directive implementation in the setting of hospital pharmacy, an assessment of European hospital pharmacies aiming to map workflows of medicines dispensation was used. Different dispensation models were analyzed, aiming for the best implementation of the directive requirements. The location of verification and decommissioning steps in different scenarios were defined. Reference models were designed, and possible variances were discussed. Workflow analysis was carried out and eight different dispensing scenarios have been identified: manual, unitdose, compounding, robotic, electronic cabinet, robotic compounding and unit-dose, and conveyor belt. Minimum one verification and decommissioning steps were necessary to maintain up-to-date information about identified packages of medicines. The use of automated systems increases the number of verifications. Models proposed support stakeholder compliance with the directive and increased patient safety by providing up-todate information about product dispensed in basic and advanced models of hospital pharmacy operations.
\end{abstract}

Keywords: decommissioning, dispensation, hospital pharmacy, Falsified Medicines Directive, verification

The Falsified Medicines Directive (FMD) (1), introduced safety features on the packages of medicines, which facilitates the verification of the authenticity of a medicine. The details of implementing a Europe-wide system were included in the Delegated Regulation (2). The regulation did not introduce any operational procedures for hospital pharmacies. Thus, different healthcare institutions implemented and evolved existing solutions to meet the requirements of the regulation. Regulatory and operational implications already affected the dispensing procedures in hospital pharmacies. In this study, we reviewed the workflow of several hospital pharmacies in Europe to design reference models of

* Corresponding author: e-mail: p.merks@uksw.edu.pl 
the verification of the authenticity of medicine in different dispensing scenarios.

Nowadays, hospital pharmacists authenticate each package, which contains the unique identifier, FMD mandated 2D data matrix. Two different processes have been distinguished: verification and decommissioning. In the verification process, the serial number of the package is queried in a national repository system and its authenticity is determined based on whether the scanned code is stored in the national repository or not. A decommission scanning process carries out the same function as verification but also decommissions the product in the national database. That ultimately confirms that the product was dispensed. Hospitals can select at what stages verification and decommission occur. From the moment the decommission, the hospital has ten days to revert the status of the unique identifier, e.g. if a product was not used. Limitation impacts the operations of a pharmacy in terms of supplying other hospitals or loans. Also, an economic impact will occur because a return to the supplier will not be possible after ten days elapse. Also, the system brings new features to benefit the hospital's daily operations. It provides additional patient safety checks along with FMD compliance notifying about recalled, expired, or suspicious medicinal products (3). Information is dynamic. Hospitals can select where to perform the decommissioning of the product. The decommission of medicines at an early stage (e.g. at goods-in) has limited value, as some products may be dispensed months or years after the initial authentication. The best practice requires a minimum of two scanning stages, the first for initial verification and the second for the decommissioning before product supply to a ward. Two scans, verification and decommissioning, instead of decommissioning only, increase scanning time and resources used (4-6). However, the more labor-consuming practice would help to avoid supplying an expired, recalled, or dangerous falsified medicine. We reviewed the number of dispensing scenarios in a variety of hospitals and made recommendations on how to adapt workflows to the regulatory implications of the FMD.

\section{EXPERIMENTAL}

The study was based on real-life dispensing scenarios identified in European hospital pharmacies before the implementation of FMD. Methods (questionnaire and direct assessment) and the results of some of these assessments were published previously (5-7). All possible workflows were mapped and the implementation of the FMD requirements was studied to offer the best utilization of benefits of the FMD compliance. For each dispensing scenario reference model of FMD implementation was developed. The study did not involve humans. The study was performed in the following hospitals: Red Cross Hospital, in Athens, Greece; Hospital Sousa Martins, Guarda, Portugal; Bajcsy-Zsilinszky Korhaz Hospital, Budapest, Hungary; Landeskrankenhaus, Innsbruck, Austria; Heidelberg University

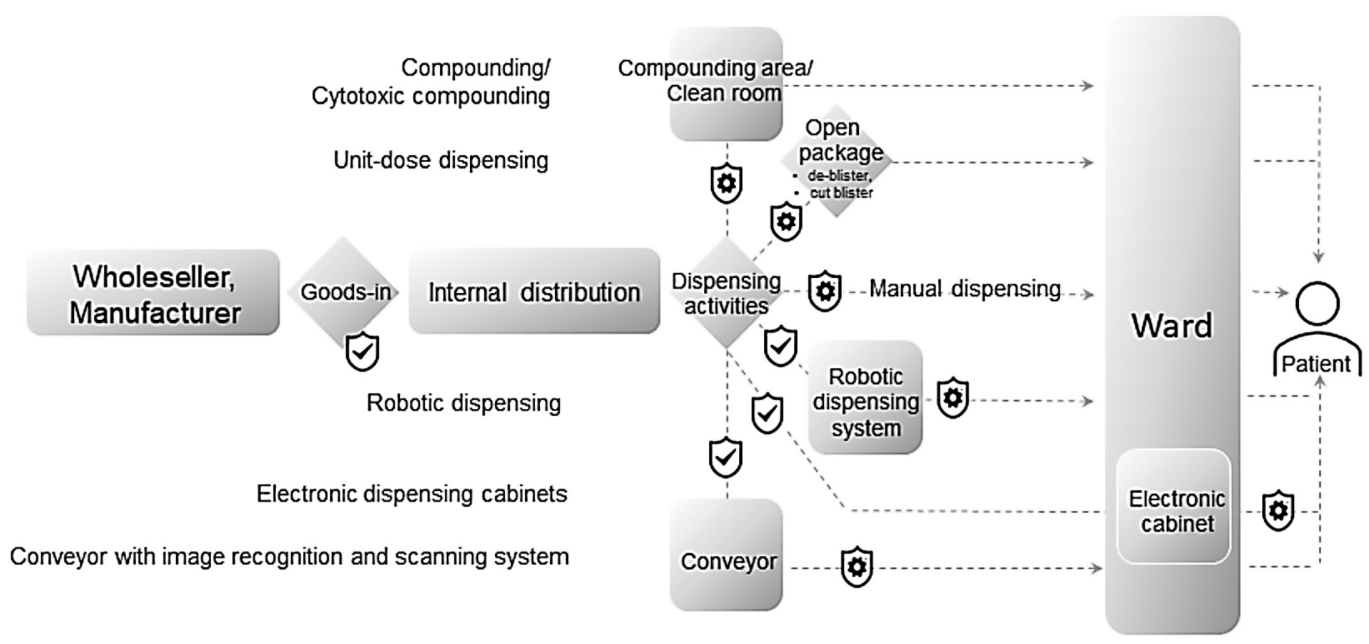

Figure 1. The Falsified Medicines Directive implementation in different models of dispensation. Typically, in one hospital more than one model is present but all need to adhere to principles of the regulation. Workflow from the delivery to a hospital to the patient is illustrated. Verification (shield with a checkmark) and decommissioning (shield with gear) steps are indicated. 
Hospital, Heidelberg, Germany; Hôpital a Bruxelles Cliniques Universitaires Saint Luc, Brussels, Belgium; Universitätsklinikum Hamburg-Eppendorf, Hamburg, Germany; University Teaching Hospital of Zemplen, in Miskolc, Hungary, and Hospital Cova da Beira, Covilha, Portugal.

\section{RESULTS AND DISCUSSION}

We identified eight main dispensing scenarios. Figure 1 illustrates the best practice of FMD-compliant workflow for each scenario; however, some deviations can be accepted depending on technical capabilities and operating procedures.

The medicinal products are received at goodsin delivered from pharmaceutical companies or wholesalers; the regular inventory management also occurs at this point. Verification of the products should be done at this location to ensure that at the time of receipt, medicines procured are not expired, recalled, or falsified (Fig. 1). The medicinal products are moved through the internal supply chain of the hospital pharmacy to various dispensing points (compounding, unit-dose, automated systems).

\section{Manual dispensing}

Manual package dispensing by pharmacy staff is the most common scenario (8). When the prescription or request is received in the hospital pharmacy, the dispensing process begins. The product is collected and the correctness of the medicine dispensed is confirmed. Typically, when the package leaves the premises of the hospital pharmacy, the decommission step occurs. This ensures compliance with the regulation by confirming that the product is safe to be dispensed. Decommissioning should occur at the last possible point in the dispensing procedure to ensure up-to-date information and reduce the impact of the ten days' rule. The setting of manual verification and decommissioning requires scanners in two locations or the use of a mobile scanner (s).

\section{Unit-dose dispensing}

Unit-dose is a method of preparing medications in which individual doses are prepared by the pharmacy and delivered in individually labeled packets to a patient. The European survey of hospital-based pharmacy services, conducted in 2000 by the European Association of Hospital Pharmacies (8), showed that unit-dose distribution became popular in Europe; $23.4 \%$ of the respondent hospitals used such systems, commonly in Belgium, Germany, and Spain.
A package of medicinal product needs to be decommissioned before breaking the anti-tampering device. In terms of workflow, the same steps are required, as in the manual dispensing scenario. The pharmacy staff would decommission the product, open the pack, and cut the blister into unit doses to be used in the dispensing process (Fig. 1)

\section{Compounding and cytotoxic compounding}

Compounding occurs in hospitals that produce medicinal products from indigents. The preparation of cytotoxic drugs requires aseptic conditions and the prevention of occupational exposure. That requires a controlled environment that's function is to prevent a process from contamination in the form of dust and airborne microbes, the cleanroom.

Some of the ingredients used in the compounding bear the safety features defined by the FDM. They need to be decommissioned in the compounding area if that is where the pack is opened the first time (Fig. 1). Before a product enters the cleanroom, it must be removed from cardboard. Thus, decommissioning should take place before entering the cleanroom (Fig. 1).

\section{Robotic package dispensing}

Robotic dispensing is being implemented in $6.7 \%$ of European hospitals in 2010 (8). Automation helps hospital pharmacies to dispense medicinal products accurately and efficiently. However, it adds further complexity for the dispensing operations under the FMD. The robot specification compliant with the FMD includes every package scanning at entry and exit and operational quarantine of negatively verified products.

During verification upon entry into the robot (Fig. 1), if an alert is generated through the authentication system, the robot needs to continue the scanning operations. The medicinal product that generated the alert needs to be excluded and put in a quarantine area to be checked by a qualified professional. When a prescription or request reaches the hospital pharmacy, the medicinal product will be dispensed through the robot. The decommissioning needs to happen at the exit (Fig. 1).

\section{Automated cabinets}

Electronic dispensing cabinets can be located everywhere in a hospital's internal supply chain. It was estimated that $10.9 \%$ of European hospitals used that method of dispensing (8). Cabinets are typically located in the ward. Decommission of medicinal products can occur in the ward before supplying to the patient (Fig. 1). Alternatively, phar- 
macists could decommission products in the hospital pharmacy, before transferring them to the electronic cabinet in the ward. The responsibility of dispensing is transferred to the ward staff.

\section{Robotic cytotoxic compounding}

Some hospitals have an automated cytotoxic compounding unit. The robot, under a strict protocol, prepares cytotoxic medicines. In such a setting, the medicinal product needs to be decommissioned before it is opened and used in the production of the cytotoxic drug. Some robots could have a storage capacity, so best practice would include a scanning point in the robot, upon entry, like robotic package dispensing.

\section{Semi-automatic dispensing with image recogni- tion using a conveyor belt}

There are pharmacies having conveyor belts, with an image recognition system incorporated with a scanner that recognizes the drug and scans code at the end of the belt. The conveyor then diverts the drug to the specific token for delivery to the relevant ward. The same operational protocols need to be in place as referred to earlier in the robotic package dispensing section (Fig. 1), i.e., there should be a defined quarantine area, and the conveyor needs to continue operating even if a product generates an alert.

\section{Unit-dose robot}

Unit-dose robots will have the same type of workflow as the manual unit-dose scenario, except for the former being automated. The hospital pharmacy staff needs to decommission the medicinal product before opening the package of the medicinal product and introducing the drugs into the unit dose robot. The robot could do the decommission process if the required hardware is present and the software will allow a connection to an authentication system. The decommission can be made through the robotic software before opening the medicinal product.

\section{CONCLUSIONS}

Typically, hospitals across Europe have more than one of the dispensing scenarios identified in the study. Each scenario needs to be analyzed individually to optimize the adoption of the FMD. Verification of authenticity should be performed as early as possible, e.g., at goods-in and products should be reverified at the entry of automated dispensing solutions. Decommissioning should be performed as late as possible to maintain the benefit of up-to-date information about the status of a product before dispensing to patients.

\section{Acknowledgments}

We wish to express our sincere thanks to the following healthcare institutions that have contributed to the development of authentication projects in the secondary care environment: Red Cross Hospital, in Athens, Greece; Hospital Sousa Martins, Guarda, Portugal; Bajcsy-Zsilinszky Korhaz Hospital, Budapest, Hungary; Landeskrankenhaus, Innsbruck, Austria; Heidelberg University Hospital, Heidelberg, Germany; Hôpital a Bruxelles Cliniques Universitaires Saint Luc, Brussels, Belgium; Universitätsklinikum Hamburg-Eppendorf, Hamburg, Germany; University Teaching Hospital of Zemplen, in Miskolc, Hungary and Hospital Cova da Beira, Covilha, Portugal.

We wish to express our sincere thanks to the healthcare institutions that have contributed to the development of authentication projects in the secondary care environment and contribute to this study. We thank Marcin Balcerzak of Medink for medical writing support.

\section{Disclosures}

The content outlined herein represents the individual opinions of the authors and may not necessarily represent the viewpoints of their employers. P.M was employed as Business Development Director of Aegate Limited at the time of preparation of this research now is employed at the Cardinal Stefan Wyszyński University in Warsaw, Poland. D.B. is a stockholder in Translation Ventures Ltd. (Charlbury, Oxfordshire, UK) and IP Asset Ventures (Oxford, UK). D.B. is subject to the CFA Institute's Codes, Standards, and Guidelines, and as such, this author must stress that this piece is provided for academic interest only and must not be construed in any way as an investment recommendation. Additionally, at time of publication, D.B. and the organizations with which he is affiliated may or may not have agreed and/or pending funding commitments from the organizations named herein.

\section{Conflicts of interest}

The authors declare no conflict of interest.

\section{REFERENCES}

1. Directive 2011/62/EU of the European Parliament and of the Council of 8 June 2011 
amending Directive 2001/83/EC on the Community code relating to medicinal products for human use, as regards the prevention of the entry into the legal supply chain of falsified medicinal products. pp. 74-87, Official J. Eur. Union (2011).

2. Commission Delegated Regulation (EU) 2016/161 of 2 October 2015 supplementing Directive 2001/83/EC of the European Parliament and of the Council by laying down detailed rules for the safety features appearing on the packaging of medicinal products for human use. pp. 1-27, Official J. Eur. Union (2015).

3. Klein K., Stolk P.: Drug Saf. 41, 911 (2018).
4. The Falsified Medicines Directive - implementing practical solutions in the hospital arena. European Alliance for Access to Safe Medicines. Essex, United Kingdom (2019).

5. Merks P., Swieczkowski D., Zerhau M., Gawronska A., Kowalczuk A., et al.: Pharmacy (Basel) 8, 1 (2020).

6. Pinto de C.N., Koshkouei M.J., Jeske M., Zeiler M., Brindley D.A.: J. Hosp. Med. Manage. 3, 1 (2017).

7. Religioni U., Swieczkowski D., Gawronska A., Kowalczuk A., Drozd M., et al.: Pharmacy (Basel) 5, 4 (2017).

8. Frontini R., Miharija-Gala T., Sykora J.: Eur. J. Hosp. Pharm. 19, 385 (2012).

(C) 2020 by Polish Pharmaceutical Society. This is an access article under the CC BY NC license 